\title{
Low serum IgG4 level: a potential diagnostic biomarker for IgA nephropathy
}

\author{
Xinyu Tian ${ }^{1}$, Zhuan Cui ${ }^{1}$, Song Wang ${ }^{1}$, Yuejuan Pan ${ }^{1}$, Lata $\mathrm{A}^{1}$, Xinxin Chen ${ }^{1}$, Xiaoxiao Wang ${ }^{2}$, \\ Xiaoyan Qiu $^{3}$, Zhenling Deng ${ }^{1}$, Yue Wang ${ }^{1}$
}

${ }^{1}$ Department of Nephrology, Peking University Third Hospital, Beijing, China; ${ }^{2}$ Research Center of Clinical Epidemiology, Peking University Third Hospital, Beijing, China; ${ }^{3}$ Department of Immunology, Peking University Health Science Center, Beijing, China

Contributions: (I) Conception and design: Y Wang, Z Deng, X Tian; (II) Administrative support: Y Wang, Z Deng; (III) Provision of study materials or patients: X Tian, Z Cui, S Wang, Y Pan, L A, X Chen, X Qiu; (IV) Collection and assembly of data: X Tian, Z Deng; (V) Data analysis and interpretation: X Tian, X Wang; (VI) Manuscript writing: All authors; (VII) Final approval of manuscript: All authors.

Correspondence to: Yue Wang; Zhenling Deng. Department of Nephrology, Peking University Third Hospital, 49 Huayuanbei Road, Beijing 100191, China. Email: bjwangyue@sina.com; dengzhenling1985@126.com.

Background: In contrast to intense investigations of galactose-deficient immunoglobulin A (IgA)1 specific
immunoglobulin G (IgG), little is known about the IgG subclasses in IgA nephropathy (IgAN). Low IgG4
levels in IgAN were noticed in our preliminary experiment. We aimed to verify the low IgG4 levels and
investigate the related immune mechanism in IgAN.
Methods: A total of 112 healthy controls (HC) and 112 newly diagnosed IgAN patients were enrolled
in this study. Patients with idiopathic membranous nephropathy (IMN), minimal change disease (MCD),
or lupus nephritis (LN) were selected as disease controls (DC) (n=122). Serum IgG4 and IgG levels were
detected by enzyme-linked immunosorbent assay (ELISA). The IgG4 ${ }^{+}$B, T helper 1 (Th1), and Th2 cells
were measured by flow cytometry. Receiver operating characteristic curves (ROC) were performed to
evaluate the diagnostic value of IgG4. Results: Both IgG4 levels and IgG4/IgG in IgAN were lower than HC and DC (all $\mathrm{P}<0.001)$. Severe IgAN displayed lower IgG4 levels than mild IgAN (P=0.039). Patients with higher risk of renal progression $(>50 \%)$ demonstrated lower IgG4 levels than lower-risk $(\leq 15 \%)$ patients $(\mathrm{P}=0.019)$. The cutoff value of $\operatorname{IgG} 4$ in differentiating IgAN from $\mathrm{HC}$ and DC was $0.26 \mathrm{mg} / \mathrm{mL}$ [sensitivity $98.2 \%$, specificity $82.4 \%$, area under the curve (AUC): $0.941, \mathrm{P}<0.0001]$ and $0.17 \mathrm{mg} / \mathrm{mL}$ (sensitivity $90.2 \%$, specificity $85.2 \%$, AUC: 0.937 , $\mathrm{P}<0.0001$ ), respectively. IgG4/IgG displayed similar diagnostic and differential ability. The $\operatorname{IgG} 4^{+} \mathrm{B} / \mathrm{B}$ cells $(\mathrm{P}<0.0001)$ and Th2/Th $(\mathrm{P}=0.042)$ of IgAN were lower than HC.

Conclusions: Serum IgG4 levels were low in IgAN. Lower IgG4 levels indicated more severe disease conditions and higher risk of renal progression. Low serum IgG4 seemed to be a potential diagnostic biomarker for IgAN. Decreased IgG4 ${ }^{+}$B cells and Th2 cells may contribute to the low IgG4 levels in IgAN.

Keywords: IgA nephropathy (IgAN); immunoglobulin G4 (IgG4); biomarker; B lymphocytes; T helper 2 cells (Th2 cells)

Submitted Oct 20, 2020. Accepted for publication Feb 04, 2021.

doi: 10.21037/atm-20-7007

View this article at: http://dx.doi.org/10.21037/atm-20-7007

\section{Introduction}

As the most common type of primary glomerulonephritis (GN) worldwide, IgA nephropathy (IgAN) is the leading cause of end-stage renal disease (ESRD) in young people (1). It is widely accepted that the galactosedeficient immunoglobulin A1 (Gd-IgA1) and its specific immunoglobulin $\mathrm{G}(\mathrm{IgG})$ antibodies are involved in the pathogenesis of $\operatorname{IgAN}(2)$. In contrast to intense 
investigations of Gd-IgA1-specific IgG, little is known about the IgG subclasses in IgAN. To explore the serum IgG subclass levels in IgAN, we conducted a preliminary experiment and found that the IgG4 levels of IgAN participants were significantly lower than healthy control (HC) and idiopathic membranous nephropathy (IMN) participants (Figure S1).

Among the 4 IgG subclasses, IgG4 has unique antiinflammatory potential. As a result of the half molecule exchange, which is the most peculiar feature of IgG4, a considerable proportion of IgG4 molecules are bispecific and monovalent rather than traditional bivalent antibodies, causing their inability to form large IgG4-associated immune complexes (3). Furthermore, IgG4 cannot activate complement, and it can suppress the other IgG subclassmediated complement activation (4). The immune complex (IC) containing Gd-IgA1 in IgAN can promote the proliferation of human mesangial cells (5), while IgG4 has been reported to inhibit IC formation through preventing cross-linking of antigens in autoimmune myasthenia gravis (MG) (3). The activation of complement can further aggravate the proliferation of mesangial cells and excessive production of inflammatory mediators in $\operatorname{IgAN}(6)$, whereas IgG4 has been demonstrated to suppress complement activation by competing with $\operatorname{IgG} 1$ or IgG3 for similar antigens in bullous pemphigoid (7). The relevance between IgG4 and IgAN is noteworthy on account of the IC formation and complement activation, which can be inhibited by IgG4, which are important factors in the pathogenesis of IgAN (8).

As with elevated IgG4 levels, which have received a great deal of attention in IgG4 related diseases, diseases associated with low serum IgG4 levels (summarized in our previous review) are not uncommon (9). Infection, especially recurrent respiratory infection, is commonly related to low IgG4 levels and the most common risk factor for IgAN (10). Thus, it is intriguing whether there is a possible relationship between low IgG4 levels and IgAN.

IgG4 are produced by B cells and plasma cells. T helper 2 (Th2) and its related interleukin-4 (IL-4) and interleukin-13 (IL-13) induce a class switch to IgG4 (11), whereas Th1 and its cytokines promote B cells produce complementfixing IgG subclasses (12). Therefore, measurement of Th1 and Th2 in the peripheral blood samples of IgAN patients may be conducive to exploring the immune mechanism of low IgG4 levels. This research aimed to verify low IgG4 levels and the related immune mechanism in IgAN patients. We present the following article in accordance with the
STROBE reporting checklist (available at http://dx.doi. org/10.21037/atm-20-7007).

\section{Methods}

\section{Study population}

This study included 112 newly diagnosed and treatmentnaive IgAN patients. The diagnosis of IgAN is made via kidney biopsy and is defined as dominant mesangial $\operatorname{IgA}$ deposits with the proliferation of mesangial cells and enlargement of mesangial matrixes. Blood samples were collected with a kidney biopsy. The exclusion criteria were as follows: diseases that may affect the renal function or immunoglobulin levels such as diabetes, hepatitis B or C, liver cirrhosis, systemic lupus erythematosus, hyperthyroidism, Sjogren syndrome, allergic diseases, plasma cell dyscrasia, and acquired immune deficiency syndrome; infection occurred within 3 months before the enrollment; corticosteroid or immunosuppressive therapy before kidney biopsy; unavailable blood samples.

The $112 \mathrm{HC}$ were matched with the IgAN group for gender and age. All HC had a negative urine test for erythrocytes and proteins. To study whether the low IgG4 levels were specific and able to discriminate IgAN from other glomerulonephritides, we selected biopsy-proven patients with IMN ( $\mathrm{n}=71)$, minimal change disease (MCD) $(\mathrm{n}=34)$, or lupus nephritis $(\mathrm{LN})(\mathrm{n}=17)$ as disease controls (DC) $(\mathrm{n}=122)$. All patients and controls were recruited in the Peking University Third Hospital. This study was approved by the Medical Ethics Committee of Peking University Third Hospital (M2017379) and was conducted following the Declaration of Helsinki (as revised in 2013). All participants signed the informed consent before inclusion.

\section{Clinical data collection}

The clinical data of participants were collected at the time of kidney biopsy, including gender, age, blood pressure, serum creatinine ( $\mathrm{SCr}$ ), 24-hour urine protein excretion (24h-UP), estimated glomerular filtration rate (eGFR), the Oxford scores of pathologic lesions, and medications use at or prior to biopsy. The eGFR was computed using the Chronic Kidney Disease Epidemiology Collaboration (CKD-EPI) two-level race equation (13). Hypertension was defined as systolic pressure $\geq 140 \mathrm{mmHg}$ or diastolic pressure $\geq 90 \mathrm{mmHg}$ at rest or the use of anti-hypertension drugs. 


\section{Measurement of disease severity and risk prediction}

To make comprehensive assessment of the disease severity of IgAN, we selected 3 factors to divide the IgAN participants into mild and severe groups $(14,15)$. Mild IgAN should meet the following 3 conditions: eGFR $\geq 60 \mathrm{~mL} / \mathrm{min} / 1.73 \mathrm{~m}^{2}$, $24 \mathrm{~h}-\mathrm{UP}<1 \mathrm{~g} /$ day, MEST $<4$. Severe IgAN should meet the following 3 conditions: eGFR $<60 \mathrm{~mL} / \mathrm{min} / 1.73 \mathrm{~m}^{2}, 24 \mathrm{~h}$ $\mathrm{UP} \geq 1 \mathrm{~g} /$ day, MEST $\geq 4$. The risk of renal progression 60 months after renal biopsy was calculated by QxMD according to the International IgAN Prediction Tool which was based on the age, blood pressure, eGFR, 24h-UP, Oxford scores, and medications use at or prior to biopsy of each participants (16). The web-based calculator was available at https://qxmd.com/calculate-byqxmd. Renal progression was defined as a $50 \%$ decline in eGFR or ESRD.

\section{Collection of blood samples}

The serum of participants was separated by centrifugation and stored at $-80{ }^{\circ} \mathrm{C}$ until the time of testing. Peripheral blood mononuclear cells (PBMCs) were separated from whole blood using the human lymphocyte separation medium (Dakewe, 7111011, Shenzhen, China) through density gradient centrifugation. The PBMCs were stored in liquid nitrogen until Th1 and Th2 detection.

\section{Laboratory measurements}

The serum concentrations of IgG4 and IgG were measured using enzyme-linked immunosorbent assay (ELISA) kits (IgG4: Cat\# BMS2095, RRID: AB_2575419, Thermo Fisher Scientific; IgG: Cat\# NBP2-60474, RRID: AB_2876878, Novus) according to the manufacture's instructions. The IgG4/IgG ratio was calculated.

IgG4 positive B cells were detected using flow cytometry in the fresh whole blood of $35 \mathrm{HC}$ and 35 IgAN participants (gender and age-matched). Anticoagulated [with ethylenediamine tetraacetic acid (EDTA)] peripheral venous blood $(150 \mu \mathrm{L})$ was surface-stained with a fluorescein isothiocyanate (FITC)-conjugated anti-CD19 antibody (Cat\# 11-0199-41, RRID: AB_10668005; Thermo Fisher Scientific, Waltham, MA, USA). Then, the sample was incubated for 30 minutes at $4{ }^{\circ} \mathrm{C}$ in the dark. After being washed with phosphate-buffered saline (PBS), the sample was fixed and permeabilized using the transcription factor fixation/permeabilization buffer set (Biolegend, 424401,
San Diego, CA, USA). Finally, intracellular staining with an allophycocyanin (APC)-conjugated anti-IgG4 antibody (Cat\# CYT-IGG4AP, RRID: AB_2876879; Cytognos, Salamanca, Spain) was performed.

The Th1 and Th2 cells were detected in PBMCs of $36 \mathrm{HC}$ and $35 \mathrm{IgAN}$ participants (gender and age-matched) using flow cytometry. The PBMCs that were resuspended in Roswell Park Memorial Institute (RPMI) 1640 medium (11875093, Gibco, Waltham, MA, USA) were incubated in the 24-well microplates (3524, Corning, NY, USA). A pre-mixed cocktail with an optimized concentration of PMA (phorbol 12-myristate-13-acetate) and ionomycin (Biolegend, 423301) was added to stimulate the cells. Subsequently, $1 \times$ brefeldin A (Biolegend, 420601) was supplemented. After incubation at $37{ }^{\circ} \mathrm{C}$ in $5 \% \mathrm{CO}_{2}$ for 6 hours, cells were surface-stained with PerCPCy5.5conjugated anti-CD3 (BioLegend Cat\# 317335, RRID: AB_2561627) and FITC-conjugated anti-CD8 (BioLegend Cat\# 301005, RRID: AB_314123) antibodies. Intracellular staining with $\mathrm{PE}$-conjugated anti-interferon (IFN) $\gamma$ (BioLegend Cat\# 502509, RRID: AB_315234) and APCconjugated anti-IL-4 antibodies (BioLegend Cat\# 500713, RRID: AB_1877160) was performed after fixation and permeabilization using the fix and perm kit (GAS003, Multi Sciences, Hangzhou, China).

Cell acquisition was performed using a BD FACSVerse cytometer (BD Biosciences, San Jose, CA, USA). The ratio of IgG4 ${ }^{+} \mathrm{B}$ cells over total B cells was calculated. The Th cells were defined as $\mathrm{CD}^{+}{ }^{+} \mathrm{CD} 8^{-}, \mathrm{IFN}-\gamma^{+} \mathrm{IL}_{-} 4^{-} \mathrm{Th} 1$ cells and IL- $4^{+}$IFN $\gamma^{-}$Th2 cells were subsequently defined. The ratios of Th1/Th, Th2/Th, and Th1/Th2 were calculated. Data of flow cytometry were analyzed using the Kaluza Analysis version 2.1 (RRID:SCR_016182, Beckman Coulter, Kaluza, Brea, CA, USA).

\section{Statistical analysis}

Based on our pilot study (including 58 IgAN participants, $56 \mathrm{HC}$, and $51 \mathrm{DC}$ ), which generated a sensitivity $\geq 0.93$ and specificity $\geq 0.75$ in the diagnostic test, the minimum sample size calculated by the PASS 11 software [Power Analysis and Sample Size software, Number Cruncher Statistical System (NCSS), LLC, Kaysville, UT, USA, RRID:SCR_019099] was 63 for each group when the sensitivity and specificity were set at 0.8 . Thus, it was considered adequate to have at least 112 individuals in each group to perform the final diagnostic test. Normally distributed variables were presented as mean \pm standard deviation $(\mathrm{SD})$, and non- 
Table 1 Demographic and clinical features of subjects

\begin{tabular}{lccc}
\hline Characteristics & $\operatorname{lgAN}(\mathrm{n}=112)$ & Healthy controls $(\mathrm{n}=112)$ & ${\text { Disease controls }(\mathrm{n}=122)^{\dagger}}^{\dagger}$ \\
\hline Gender, male $(\%)$ & $51(45.54)$ & $57(50.89)$ & $74(66.66)$ \\
Age (years) & $32(28.0,39.0)$ & $33(29.0,39.0)$ & $36(27.0,51.0)$ \\
Proteinuria $(\mathrm{g} / \mathrm{day})^{\ddagger}$ & $1.58(0.88,2.65)$ & - & $6.24(3.05,9.70)$ \\
Serum creatine $(\mu \mathrm{mol} / \mathrm{L})$ & $94(71.0,130.0)$ & $78 \pm 13$ & $71(61.0,87.0)$ \\
eGFR $\left(\mathrm{mL} / \mathrm{min} / 1.73 \mathrm{~m}^{2}\right)$ & $80 \pm 29$ & $101 \pm 12$ & $102(88.0,116.0)$ \\
\hline
\end{tabular}

Values are expressed as mean $\pm \mathrm{SD}$ or median (IQR). ${ }^{\dagger}$, disease controls include patients with idiopathic membranous nephropathy (IMN, $n=71$ ), lupus nephritis (LN, $n=17$ ), and minimal change disease (MCD, $n=34) .{ }^{\ddagger}$, all healthy controls included in this study have a negative urine test for proteins and erythrocytes. IgAN, immunoglobulin A nephropathy.

A

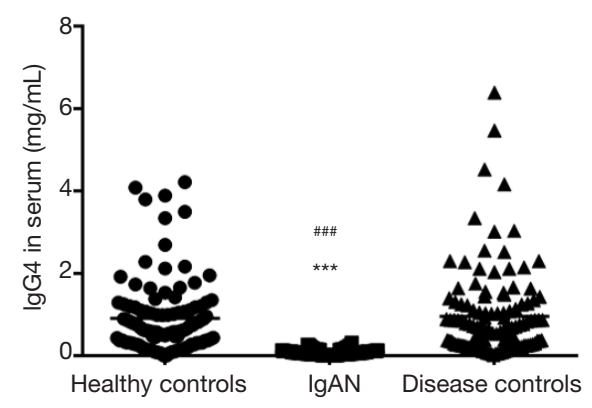

B

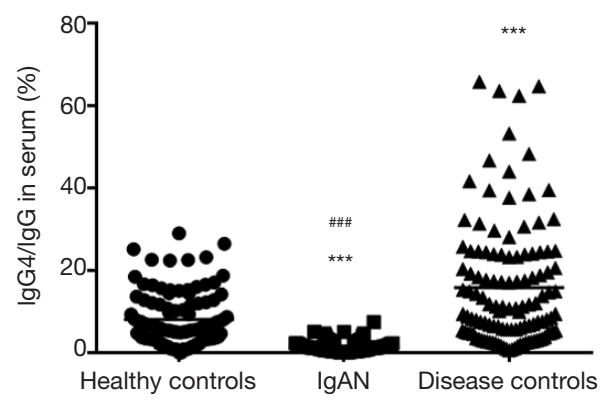

Figure 1 Comparison of IgG4 concentrations (A) and IgG4/IgG ratio (B) in different groups. The IgG4 concentrations and IgG4/IgG are significantly lower in IgAN compared with $\mathrm{HC}$ and DC. ${ }^{* *}, \mathrm{P}<0.001$, indicates comparison with healthy controls. There is no significant difference in IgG4 concentrations between HC and DC. ${ }^{\# \#}, \mathrm{P}<0.001$, indicates comparison with DC. DC, disease controls; HC, healthy controls; IgAN, immunoglobulin A nephropathy.

normally distributed variables were presented as median [interquartile range (IQR)]. Differences between groups were analyzed using $t$-test in normally distributed groups or Mann-Whitney $U$ test in non-normally distributed groups. Receiver operating characteristic (ROC) curves were performed to evaluate IgG4 and IgG4/IgG's diagnostic value. Data analyses were performed using the software SPSS 22.0 (IBM, Armonk, NY, USA; RRID:SCR_002865). The difference was considered statistically significant when a two-tailed $\mathrm{P}<0.05$. Graphs were generated using GraphPad Prism 5.0 (GraphPad, San Diego, CA, USA; RRID:SCR_002798).

\section{Results}

\section{IgG4 levels were low in IgAN participants}

A total of 346 participants were included in the detection of serum IgG4 and IgG. Sample sizes in IgAN, HC, and DC were respectively 112,112 , and 122 . Table 1 summarizes the gender, age, proteinuria, $\mathrm{SCr}$, and eGFR of participants.

As described in Figure 1A, the serum IgG4 levels $(\mathrm{mg} / \mathrm{mL})$ in IgAN $[0.08(0.04,0.12)]$ were significantly lower than those of HC $[0.61(0.32,1.16), \mathrm{P}<0.001]$ and DC $[0.68(0.26$, 1.24), $\mathrm{P}<0.001]$. The IgG4 levels were comparable between $\mathrm{HC}$ and DC. Similar results were obtained when IgG4/IgG was analyzed in the same participants and controls, except that the IgG4/IgG (\%) in DC $[10.76(4.77,23.83)]$ was higher than HC $[5.97(3.33,12.10), \mathrm{P}<0.001]$ (Figure $1 B$ ).

Table 2 shows IgG4 concentrations and IgG4/IgG in IgAN participants with different severity. Although there was no significant difference in IgG4 levels and IgG4/IgG (\%) when stratifying IgAN participants by hypertension, proteinuria, eGFR, and Oxford score separately, after comprehensive assessment of disease severity based on the combination of proteinuria, eGFR, and Oxford score, severe 
Table 2 IgG4 concentrations and IgG4/IgG in IgAN patients with different severity

\begin{tabular}{|c|c|c|c|c|c|}
\hline Group & Sample size & $\operatorname{lgG} 4(\mathrm{mg} / \mathrm{mL})$ & $\mathrm{P}^{\dagger}$ & $\operatorname{lgG} 4 / \operatorname{lgG}(\%)$ & $\mathrm{P}^{\ddagger}$ \\
\hline With & 49 & $0.07(0.04,0.13)$ & & $1.22(0.51,2.30)$ & \\
\hline Without & 63 & $0.08(0.04,0.12)$ & & $1.25(0.54,2.04)$ & \\
\hline Proteinuria (g/day) & & & 0.389 & & 0.601 \\
\hline$\geq 1$ & 81 & $0.07(0.04,0.12)$ & & $1.09(0.53,2.30)$ & \\
\hline eGFR $\left(\mathrm{mL} / \mathrm{min} / 1.73 \mathrm{~m}^{2}\right)$ & & & 0.862 & & 0.309 \\
\hline$<60$ & 27 & $0.07(0.04,0.12)$ & & $1.35(0.50 .2 .50)$ & \\
\hline$\geq 60$ & 85 & $0.08(0.04,0.12)$ & & $1.17(0.53,1.95)$ & \\
\hline M1 & 89 & $0.07(0.04,0.11)$ & & $1.13(0.53,2.11)$ & \\
\hline E0 & 36 & $0.07(0.03,0.12)$ & 0.794 & $1.24(0.38,2.07)$ & 0.827 \\
\hline $\mathrm{E} 1$ & 76 & $0.08(0.04,0.12)$ & & $1.21(0.54,2.11)$ & \\
\hline so & 32 & $0.10(0.04,0.14)$ & 0.185 & $1.42(0.72,2.17)$ & 0.285 \\
\hline S1 & 80 & $0.07(0.04,0.11)$ & & $1.10(0.50,2.10)$ & \\
\hline $\mathrm{T} \leq 1$ & 56 & $0.06(0.03,0.12)$ & 0.154 & $1.25(0.39,1.54)$ & 0.256 \\
\hline $\mathrm{T} 2$ & 56 & $0.08(0.05,0.13)$ & & $1.21(0.65,2.49)$ & \\
\hline $\mathrm{CO}$ & 59 & $0.07(0.03,0.12)$ & 0.868 & $1.34(0.59,2.11)$ & 0.543 \\
\hline Severe IgAN & 14 & $0.06 \pm 0.03$ & & $1.03 \pm 0.77$ & \\
\hline Risk of renal progression ${ }^{\S}$ & & & $0.019^{*}$ & & 0.251 \\
\hline$\leq 15 \%$ & 19 & $0.11 \pm 0.07$ & & $1.74 \pm 1.37$ & \\
\hline$>50 \%$ & 22 & $0.07 \pm 0.04$ & & $1.32 \pm 0.94$ & \\
\hline
\end{tabular}

Values are expressed as median (IQR) or mean \pm standard deviation (SD). ${ }^{\dagger}$, comparison of IgG4 between groups. ${ }^{\ddagger}$, comparison of $\mathrm{lgG} 4 / \mathrm{lgG}(\%)$ between groups. ${ }^{\S}$, the risk of renal progression 60 months after renal biopsy was calculated by QxMD according to the International IgAN Prediction Tool (16). *, P<0.05. IgAN, immunoglobulin A nephropathy.

IgAN displayed lower IgG4 levels $(\mathrm{mg} / \mathrm{mL})$ than mild IgAN $[(0.06 \pm 0.03)$ vs. $(0.10 \pm 0.05), \mathrm{P}=0.039]$. Additionally, participants with higher risk $(>50 \%)$ of renal progression demonstrated lower IgG4 levels $(\mathrm{mg} / \mathrm{mL})$ than participants with lower risk $(\leq 15 \%)$ of progression $[(0.07 \pm 0.04) v s$. $(0.11 \pm 0.07), \mathrm{P}=0.019]$. IgG4/IgG (\%) level were comparable between participants with different severity and risk.

\section{IgG4 displayed efficient diagnostic and differential ability} for $\operatorname{Ig} A N$

The ROC curve analyses showed that a cutoff value of 
A

IgAN vs. Healthy controls IgG4



C

IgAN vs. Disease controls IgG4

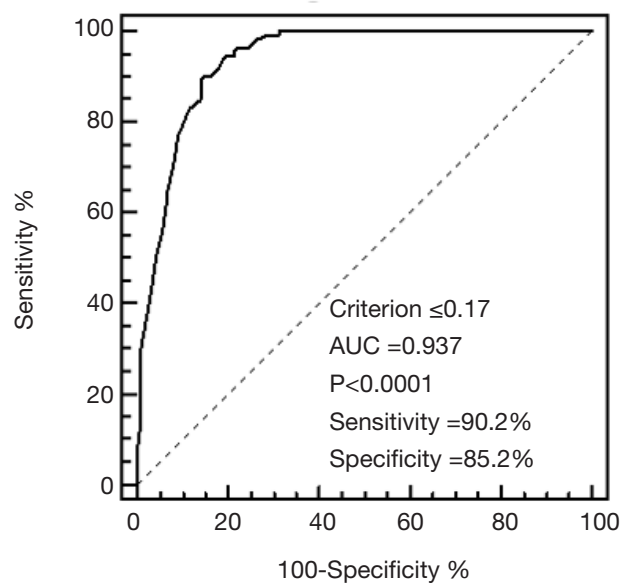

B

$\lg \mathrm{AN}$ vs. Healthy controls $\lg \mathrm{G} 4 / \lg \mathrm{G}$

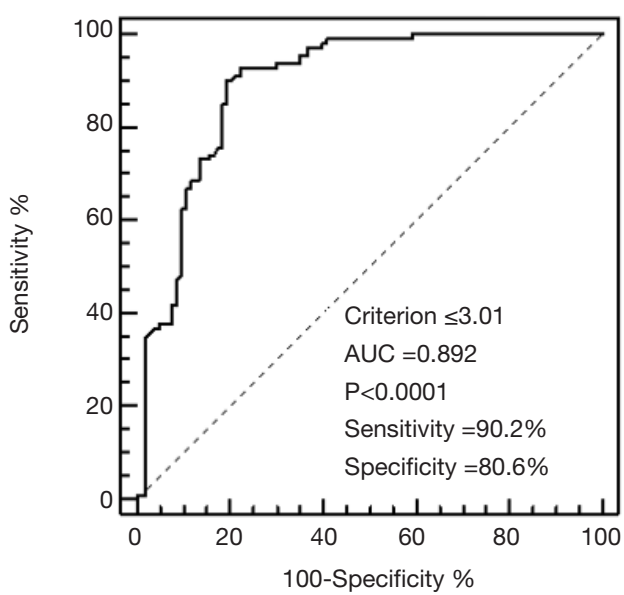

D



Figure 2 Diagnostic performance of $\operatorname{IgG} 4(\mathrm{~A}, \mathrm{C})$ and $\operatorname{IgG} / / \operatorname{IgG}(\mathrm{B}, \mathrm{D})$ in $\operatorname{IgAN}$. (A and B) reflect the ability in differentiating IgAN from HC, while (C and D) indicate the differential diagnostic ability of IgAN and its DC. Both IgG4 and IgG4/IgG displayed an efficient diagnostic and differential ability for IgAN. DC, disease controls; HC, healthy controls; IgAN, immunoglobulin A nephropathy; AUC, area under the curve.

$0.26 \mathrm{mg} / \mathrm{mL}$ for serum IgG4 (Figure $2 A$ ) and $3.01 \%$ for serum IgG4/IgG (Figure $2 B$ ) had the highest accuracy in differentiating IgAN from healthy individuals [IgG4: sensitivity $98.2 \%$, specificity $82.4 \%$, area under the curve $(\mathrm{AUC})=0.941, \mathrm{P}<0.0001 ; \mathrm{IgG} 4 / \mathrm{IgG}$ : sensitivity $90.2 \%$, specificity $80.6 \%, \mathrm{AUC}=0.892, \mathrm{P}<0.0001]$. The best cutoff value of $0.17 \mathrm{mg} / \mathrm{mL}$ for serum IgG4 (Figure 2C) and $3.27 \%$ for IgG4/IgG (Figure 2D) had the capacity to differentiate IgAN from DC with a sensitivity of $90.2 \%$ and $92.9 \%$, respectively, specificity of $85.2 \%$ and $81.7 \%$, respectively, and the AUCs of $0.937(\mathrm{P}<0.0001)$ and $0.944(\mathrm{P}<0.0001)$. According to the AUC, serum IgG4 performed better than IgG4/IgG in the diagnosis of IgAN, but displayed a similar statistic power in differentiating IgAN from DC.

\section{Decreased IgG4 $4^{+} B$ and Th2 cells in IgAN}

Compared with HC ( $\mathrm{n}=35$ for $\mathrm{B}, \mathrm{n}=36$ for $\mathrm{Th})$, the $\mathrm{IgG} 4^{+} \mathrm{B} /$ B cells (\%) ( $\mathrm{n}=35)$ and Th2/Th cells $(\%)(\mathrm{n}=35)$ in the peripheral blood of IgAN were significantly lower $[0.30$ 
A

Healthy control

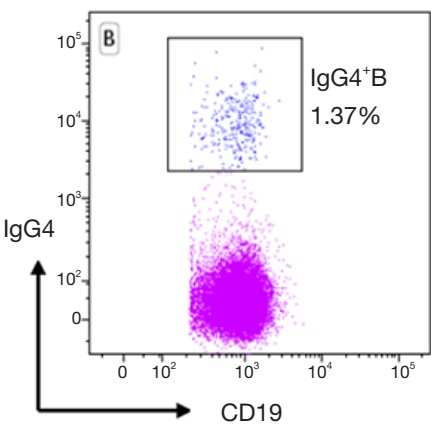

C

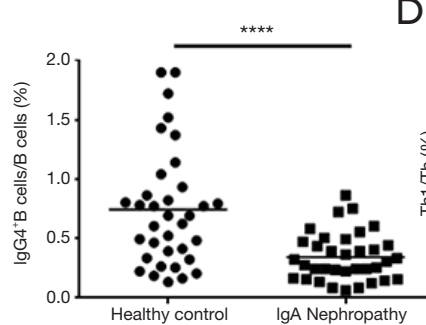

$\mathrm{D}$ $\lg \mathrm{AN}$
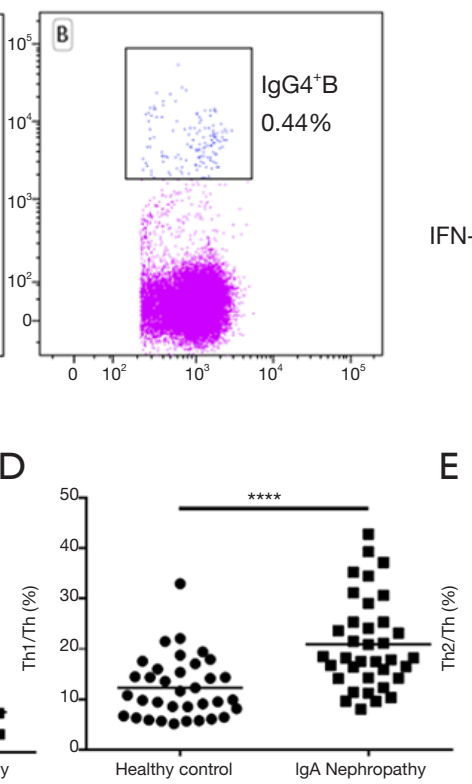

B

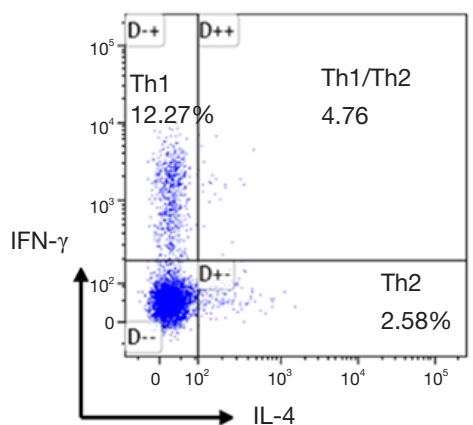

$\lg \mathrm{AN}$

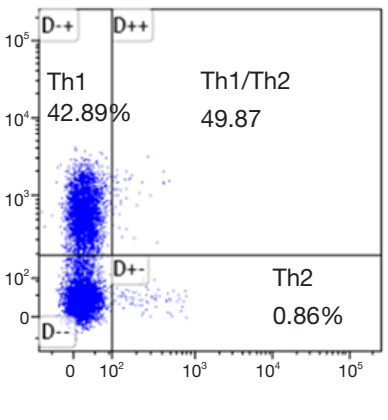

$\mathrm{E}$

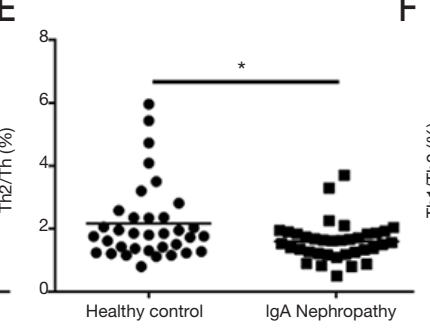

$\mathrm{F}$

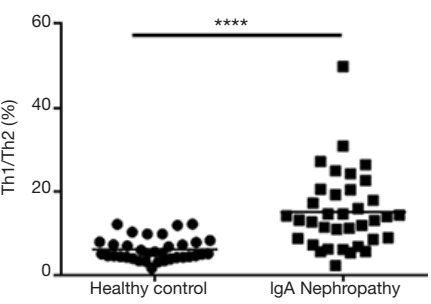

Figure 3 The IgG4 $4^{+}$B cells (A,C), Th1 cells (B,D), and Th2 cells (B,E) in the peripheral blood of HC and IgAN. (A and C) The percentage of $\mathrm{IgG}^{+} \mathrm{B} / \mathrm{B}$ cells in IgAN is lower than that of the HC. (B and D) The Th1/Th ratio in IgAN is higher than that of the HC. (B and E) The Th2/Th ratio in IgAN is lower than that of the HC. (B and F) The Th1/Th2 ratio in IgAN is higher than that of the HC. *, $\mathrm{P}<0.05 ;{ }^{* * * *}$, $\mathrm{P}<0.0001$. IgAN, immunoglubulin A nephropathy; HC, healthy controls.

(0.16, 0.47) vs. $0.69(0.33,0.93), \mathrm{P}<0.0001 ; 1.58(1.22,1.85)$ vs. 1.80 (1.30, 2.36), $\mathrm{P}=0.042]$, while the Th1/Th cells (\%) and Th1/Th2 cells were significantly higher [18.21 (14.20, 25.37) vs. 10.78 (6.65, 15.98), $\mathrm{P}<0.0001 ; 13.69(8.74,20.20)$ vs. 5.26 (4.44, 7.99), $\mathrm{P}<0.0001]$ (Figure 3).

\section{Discussion}

To our knowledge, studies focusing on the serum IgG4 levels of IgAN have scarcely been reported. In the present study, significant low serum IgG4 levels and IgG4/IgG ratio were observed in IgAN patients. IgAN patients with more severe conditions and higher risk of progression displayed lower IgG4 levels than mild and lower-risk patients. The IgG4 and IgG4/IgG showed a modest diagnostic ability in discriminating IgAN from $\mathrm{HC}$ and DC such as IMN, MCD or LN. Immune dysregulation, including decreased $\operatorname{IgG} 4^{+} \mathrm{B}$ and Th2 cells, may contribute to the low IgG4 levels in IgAN.

The reference interval of serum IgG4 in adults has been recommended as $0.052-1.250 \mathrm{~g} / \mathrm{L}$ with the upper limit being $0.86-1.35 \mathrm{~g} / \mathrm{L}$, and IgG4 levels over $2 \mathrm{~g} / \mathrm{L}$ have been reported in a few healthy subjects (17-19). The
IgG4 normally accounts for about $5 \%$ of total $\mathrm{IgG}$, and the percentage can reach $75 \%$ after chronic exposure to antigens $(20,21)$. The median IgG4 and IgG4/IgG levels in the present study's $\mathrm{HC}$ were consistent with previous reports. The higher IgG4/IgG of DC in which IMN accounts for $58 \%$ is not unexpected, as IMN is considered an IgG4-related disease in which serum IgG4/IgG can be over $50 \%(17,21)$. The concentrations of IgG4 fluctuate greatly among healthy individuals (19). However, the range of the serum IgG4 levels was significantly tighter for the IgAN participants than that of either the $\mathrm{HC}$ or DC, as shown by smaller SD, range, and IQR, indicating that the low IgG4 was universal and definite.

Lower serum IgG4 concentrations in patients with more severe conditions and higher risk of progression suggested a possible correlation between IgG4 and IgAN. In HenochSchönlein purpura nephritis (IgA vasculitis), which is similar to IgAN in pathological manifestations and mesangial GdIgA1 deposition (22), low serum IgG4 levels which were comparable with IgAN were also observed (Figure S2). Furthermore, Figure S3 showed a negative correlation between serum Gd-IgA1 and IgG4 levels in IgAN. These results indicated that IgG4 might be involved in the 
pathogenesis of IgAN.

The IgG1 of mice, which cannot activate complement, is similar to human IgG4 in function and Th2-dependency $(4,23)$. Mice deficient in IgG1 were reported as more vulnerable to $M G$, arthritis, and lethal renal disease under corresponding stimulations, the other IgG isotypes which can activate complement were frequently elevated in these disorders. Simultaneously, the additional injection of antigen-specific IgG1 was shown to protect mice from diseases through the inhibition of IC formation (23-25). The effect of mouse IgG1 presumably applies to human IgG4. The mesangial deposition of IC, in which Gd-IgA1 and its specific IgG were contained, and the sequential complement activation are crucial events in the pathogenesis of IgAN. Whether abundant IgG4 could prevent IC formation by competing for similar antigens in IgAN remains to be shown. Further studies focusing on the exact role of IgG4 in the pathogenesis of IgAN are required.

In IgG4-related diseases in which serum IgG4 levels are usually elevated, higher IgG4 $4^{+} \mathrm{B} / \mathrm{B}$ cells and Th2/Th1 cells have been observed, and a positive correlation between serum IgG4 levels and IgG4 ${ }^{+} \mathrm{B}$ cells has been found $(26,27)$. These results indirectly supported the decreased $\operatorname{IgG} 4^{+} \mathrm{B} / \mathrm{B}$ and Th2 cells in IgAN. Similar to our results, Esteve Cols et al. observed higher percentages of effector memory Th1 cells in the peripheral blood of IgAN patients (28). Increased expression of Th1 transcriptional factor T-bet and decreased Th2 transcriptional factor GATA3 have been displayed in IgAN patients' urine (29). However, Yang et al. showed lower Th1 cells and higher Th2 cells in IgAN (30), which conflicted with our results. The inconsistent results may be attributed to methodological differences. Firstly, we excluded patients with allergic diseases, usually characterized by higher Th2 cells and Th2-dominated inflammation (31,32). In contrast, allergic diseases were not displayed in the exclusion criteria of Yang et al. Secondly, Th was defined as $\mathrm{CD}^{+}{ }^{+} \mathrm{CD} 8^{-}$cells in the present study, but it was defined as $\mathrm{CD}^{+} \mathrm{CD}^{+}$by Yang et al. It was reported that surface CD4 expression could be interfered by PMA used during the Th cells' detection (33).

The elevated Th1/Th and Th1/Th2 in the peripheral blood of IgAN may be associated with the nature of nephritogenic immune responses. It was reported that Th1 and Th2 mediate different patterns of glomerular injury in glomerulonephritides through initiating diverse cellular immune responses. The Th1-predominant immune responses seem to be associated with proliferative lesions and crescent formation in glomeruli, while Th2 generally leads to membranous injury $(34,35)$. Higher Th1 related IFN- $\gamma$ has been reported in the kidney of Henoch-Schönlein purpura nephritis, and the staining grades were positively correlated with the urinary protein/creatinine ratio (36). In animal studies, bone marrow Th1 was elevated in nascent IgAN while Th2 was elevated in quiescent IgAN (37).

\section{Conclusions}

In summary, IgG4 levels in IgAN patients were low. Lower IgG4 levels indicated more severe disease conditions and higher risk of renal progression. Low serum IgG4 and IgG4/IgG seem to be remarkable diagnostic biomarkers for IgAN. Immune dysregulation, including decreased IgG4 $4^{+} \mathrm{B}$ cells and Th2, may contribute to the low IgG4 levels in IgAN.

\section{Acknowledgments}

We thank the Medical Research Center and the Information Management \& Big Data Center of Peking University Third Hospital for supporting our work.

Funding: This work was supported by the National Natural Science Foundation of China (81870488, 91642109, and 82070736) and the Peking University Medicine Seed Fund for Interdisciplinary Research (BMU2020MX030).

\section{Footnote}

Reporting Checklist: The authors have completed the STROBE reporting checklist (available at http://dx.doi. org/10.21037/atm-20-7007)

Data Sharing Statement: Available at http://dx.doi. org/10.21037/atm-20-7007

Peer Review File: Available at http://dx.doi.org/10.21037/ atm-20-7007

Conflicts of Interest: All authors have completed the ICMJE uniform disclosure form (available at http://dx.doi. org/10.21037/atm-20-7007). The authors have no conflicts of interest to declare.

Ethical Statement: The authors are accountable for all aspects of the work in ensuring that questions related to the accuracy or integrity of any part of the work are appropriately investigated and resolved. This study was 
approved by the Medical Ethics Committee of Peking University Third Hospital (M2017379) and was conducted following the Declaration of Helsinki (as revised in 2013). All participants signed the informed consent before inclusion.

Open Access Statement: This is an Open Access article distributed in accordance with the Creative Commons Attribution-NonCommercial-NoDerivs 4.0 International License (CC BY-NC-ND 4.0), which permits the noncommercial replication and distribution of the article with the strict proviso that no changes or edits are made and the original work is properly cited (including links to both the formal publication through the relevant DOI and the license). See: https://creativecommons.org/licenses/by-nc-nd/4.0/.

\section{References}

1. Floege J, Amann K. Primary glomerulonephritides. Lancet 2016;387:2036-48.

2. Rizk DV, Saha MK, Hall S, et al. Glomerular Immunodeposits of Patients with IgA Nephropathy Are Enriched for IgG Autoantibodies Specific for GalactoseDeficient IgA1. J Am Soc Nephrol 2019;30:2017-26.

3. van der Neut Kolfschoten M, Schuurman J, Losen M, et al. Anti-inflammatory activity of human IgG4 antibodies by dynamic Fab arm exchange. Science 2007;317:1554-7.

4. Lilienthal GM, Rahmoller J, Petry J, et al. Potential of Murine IgG1 and Human IgG4 to Inhibit the Classical Complement and Fcgamma Receptor Activation Pathways. Front Immunol 2018;9:958.

5. Novak J, Tomana M, Matousovic K, et al. IgA1-containing immune complexes in IgA nephropathy differentially affect proliferation of mesangial cells. Kidney Int 2005;67:504-13.

6. Daha MR, van Kooten C. Role of complement in IgA nephropathy. J Nephrol 2016;29:1-4.

7. Zuo Y, Evangelista F, Culton D, et al. IgG4 autoantibodies are inhibitory in the autoimmune disease bullous pemphigoid. J Autoimmun 2016;73:111-9.

8. Lai KN, Tang SC, Schena FP, et al. IgA nephropathy. Nat Rev Dis Primers 2016;2:16001.

9. Tian X, Deng Z, Wang S, et al. Basic Research and Clinical Reports Associated with Low Serum IgG4 Concentrations. Int Arch Allergy Immunol 2020;181:149-58.

10. Rollino C, Vischini G, Coppo R. IgA nephropathy and infections. J Nephrol 2016;29:463-8.

11. Lighaam LC, Rispens T. The Immunobiology of Immunoglobulin G4. Semin Liver Dis 2016;36:200-15.
12. Del Prete G. The concept of type- 1 and type- 2 helper $T$ cells and their cytokines in humans. Int Rev Immunol 1998;16:427-55.

13. Kong X, Ma Y, Chen J, et al. Evaluation of the Chronic Kidney Disease Epidemiology Collaboration equation for estimating glomerular filtration rate in the Chinese population. Nephrol Dial Transplant 2013;28:641-51.

14. Barbour SJ, Espino-Hernandez G, Reich HN, et al. The MEST score provides earlier risk prediction in lgA nephropathy. Kidney Int 2016;89:167-75.

15. Tanaka S, Ninomiya T, Katafuchi R, et al. Development and validation of a prediction rule using the Oxford classification in IgA nephropathy. Clin J Am Soc Nephrol 2013;8:2082-90.

16. Barbour SJ, Coppo R, Zhang H, et al. Evaluating a New International Risk-Prediction Tool in IgA Nephropathy. Jama Intern Med 2019;179:942-52.

17. Cortazar FB, Stone JH. IgG4-related disease and the kidney. Nat Rev Nephrol 2015;11:599-609.

18. Schauer U, Stemberg F, Rieger CH, et al. IgG subclass concentrations in certified reference material 470 and reference values for children and adults determined with the binding site reagents. Clin Chem 2003;49:1924-9.

19. Nirula A, Glaser SM, Kalled SL, et al. What is IgG4? A review of the biology of a unique immunoglobulin subtype. Curr Opin Rheumatol 2011;23:119-24.

20. Davies AM, Sutton BJ. Human IgG4: a structural perspective. Immunol Rev 2015;268:139-59.

21. Xia CS, Fan CH, Liu YY. Diagnostic performances of serum IgG4 concentration and IgG4/IgG ratio in IgG4related disease. Clin Rheumatol 2017;36:2769-74.

22. Suzuki H, Yasutake J, Makita Y, et al. IgA nephropathy and IgA vasculitis with nephritis have a shared feature involving galactose-deficient IgA1-oriented pathogenesis. Kidney Int 2018;93:700-5.

23. Huda R, Strait RT, Tuzun E, et al. IgG1 deficiency exacerbates experimental autoimmune myasthenia gravis in BALB/c mice. J Neuroimmunol 2015;281:68-72.

24. Strait RT, Thornton S, Finkelman FD. Cgamma1 Deficiency Exacerbates Collagen-Induced Arthritis. Arthritis Rheumatol 2016;68:1780-7.

25. Strait RT, Posgai MT, Mahler A, et al. IgG1 protects against renal disease in a mouse model of cryoglobulinaemia. Nature 2015;517:501-4.

26. Lighaam LC, Vermeulen E, Bleker T, et al. Phenotypic differences between IgG4+ and IgG1+ B cells point to distinct regulation of the IgG4 response. J Allergy Clin Immunol 2014;133:267-70.e1. 
27. Heeringa JJ, Karim AF, van Laar J, et al. Expansion of blood IgG4(+) B, TH2, and regulatory T cells in patients with IgG4-related disease. J Allergy Clin Immunol 2018;141:1831-43.e10.

28. Esteve Cols C, Graterol TF, Quirant SB, et al. Immunological Pattern in IgA Nephropathy. Int J Mol Sci 2020;21:1389.

29. Tsuruga K, Oki E, Aizawa-Yashiro T, et al. Potential Th1Th2 predominance in children with newly diagnosed IgA nephropathy. Acta Paediatr 2010;99:1584-6.

30. Yang L, Zhang X, Peng W, et al. MicroRNA-155-induced T lymphocyte subgroup drifting in IgA nephropathy. Int Urol Nephrol 2017;49:353-61.

31. Looman KI, van Meel ER, Grosserichter-Wagener C, et al. Associations of Th2, Th17, Treg cells, and IgA(+) memory B cells with atopic disease in children: The Generation R Study. Allergy 2020;75:178-87.

32. Shinoda K, Hirahara K, Nakayama T. Maintenance of

Cite this article as: Tian X, Cui Z, Wang S, Pan Y, A L, Chen X, Wang X, Qiu X, Deng Z, Wang Y. Low serum IgG4 level: a potential diagnostic biomarker for IgA nephropathy. Ann Transl Med 2021;9(9):781. doi: 10.21037/atm-20-7007 pathogenic Th2 cells in allergic disorders. Allergol Int 2017;66:369-76.

33. Nakamura K, Sasada T, Sono H, et al. Inhibition of protein kinase C-mediated CD4 down-regulation by oxidative stress in T lymphocytes. J Immunol 1996;157:5339-49.

34. Tipping PG, Kitching AR. Glomerulonephritis, Th1 and Th2: what's new? Clin Exp Immunol 2005;142:207-15.

35. Kawasaki Y, Suzuki J, Sakai N, et al. Evaluation of T helper-1/-2 balance on the basis of IgG subclasses and serum cytokines in children with glomerulonephritis. Am J Kidney Dis 2004;44:42-9.

36. Gülhan B, Orhan D, Kale G, et al. Studying cytokines of T helper cells in the kidney disease of IgA vasculitis (HenochSchonlein purpura). Pediatr Nephrol 2015;30:1269-77.

37. Suzuki H, Suzuki Y, Aizawa M, et al. Th1 polarization in murine IgA nephropathy directed by bone marrow-derived cells. Kidney Int 2007;72:319-27. 

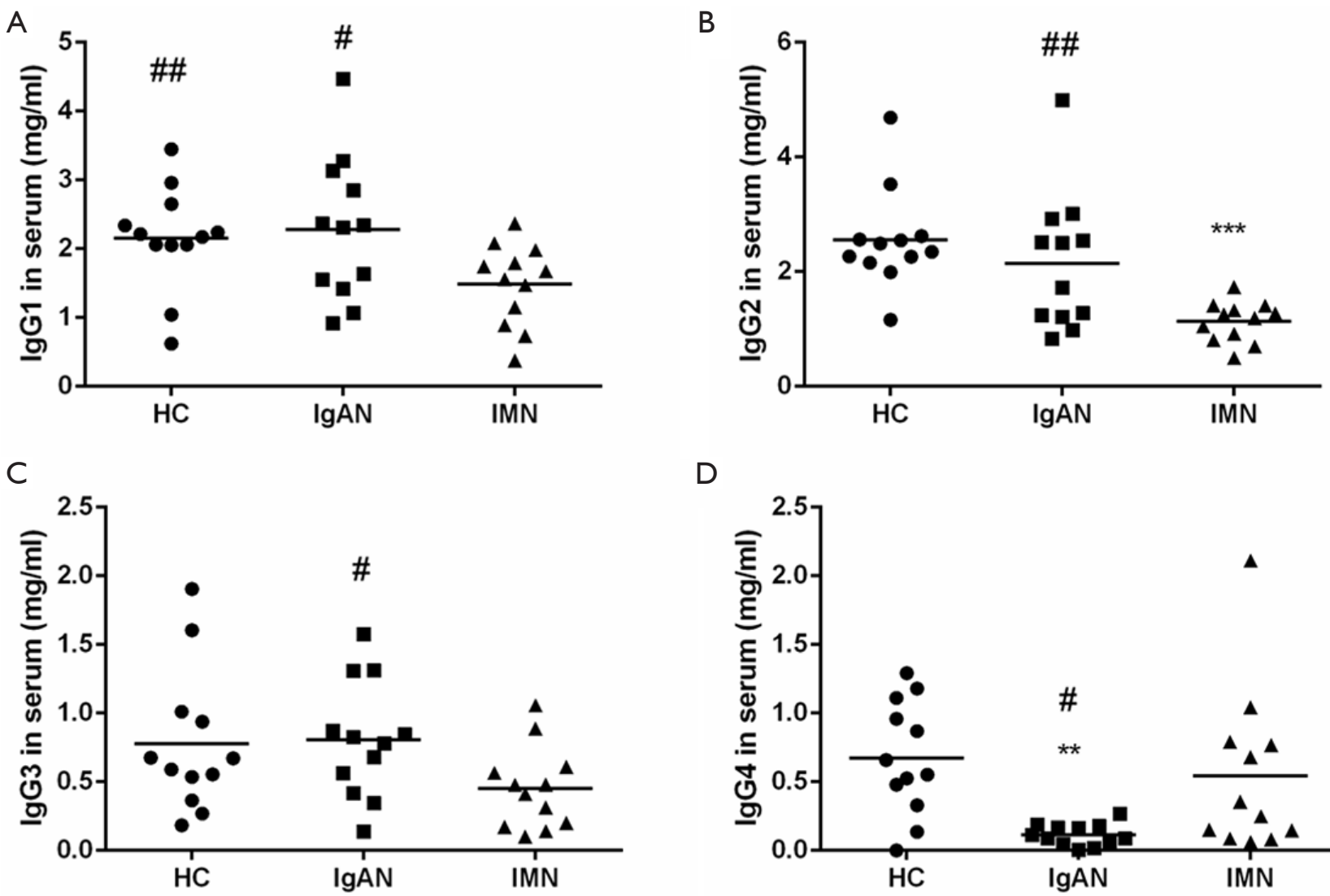

D

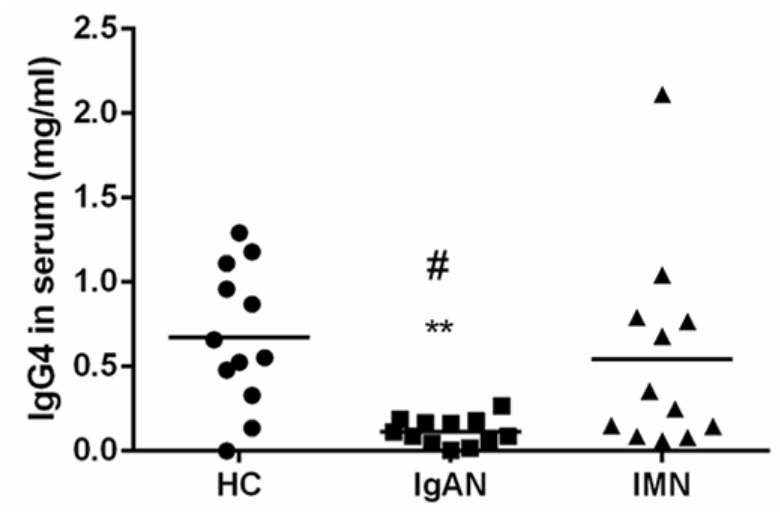

Figure S1 Comparison of IgG1, IgG2, IgG3, and IgG4 in different disease groups in preliminary experiment. (A) The serum IgG1 levels of IMN were significantly lower than HC and IgAN. (B) The serum IgG2 levels of IMN were significantly lower than HC and IgAN. (C) The serum IgG3 levels of IgAN were higher than IMN, but were comparable with HC. (D) The serum IgG4 concentrations were significantly lower in IgAN compared with HC and IMN. The sample size in each group is 12 . ${ }^{*}$, indicates comparison with $\mathrm{HC},{ }^{* *} \mathrm{P}<0.01,{ }^{* * *} \mathrm{P}<0.001$. There was no significant difference in IgG4 concentrations between HC and IMN. ${ }^{*}$ indicates comparison with IMN, ${ }^{\#} \mathrm{P}<0.05,{ }^{\#} \mathrm{P}<0.01$. HC, healthy control. IgAN, IgA nephropathy. IMN, idiopathic membranous nephropathy. 

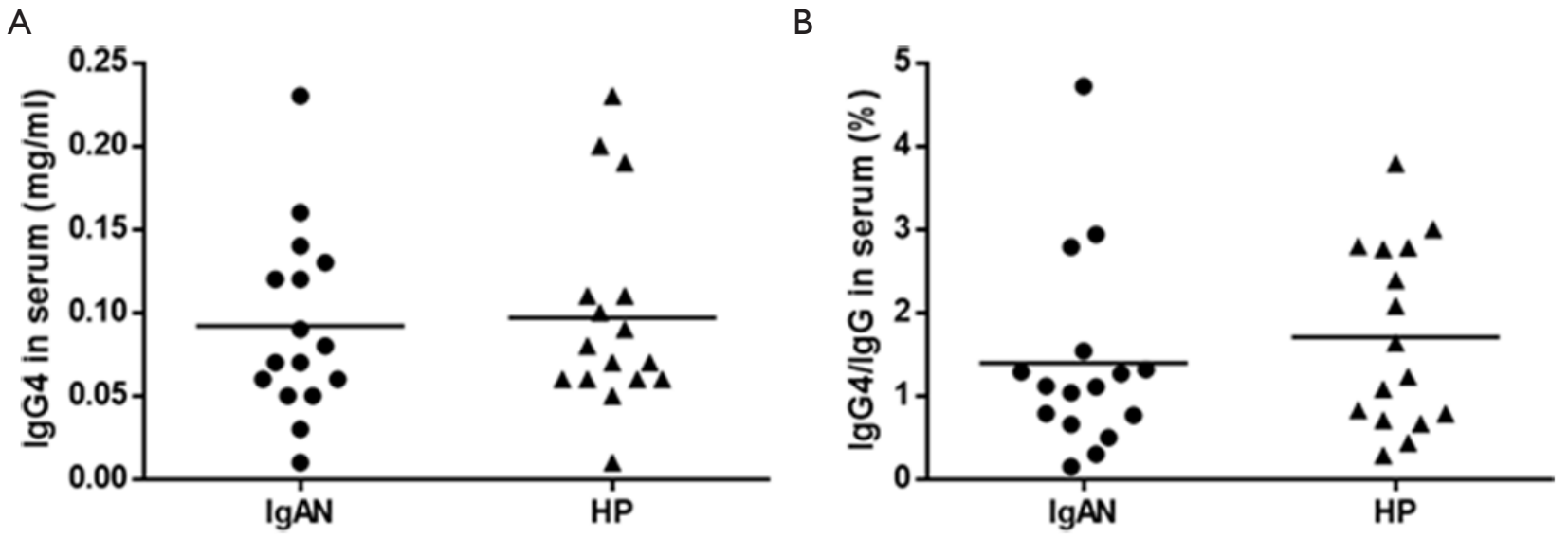

Figure S2 Comparison of IgG4 concentrations and IgG4/IgG ratio in IgAN and HP participants. The serum IgG4 (A) and IgG4/IgG (B) level in $16 \mathrm{HP}$ patients was comparable with age and gender matched IgAN patients. IgAN, IgA nephropathy. HP, Henoch-Schönlein purpura nephritis.
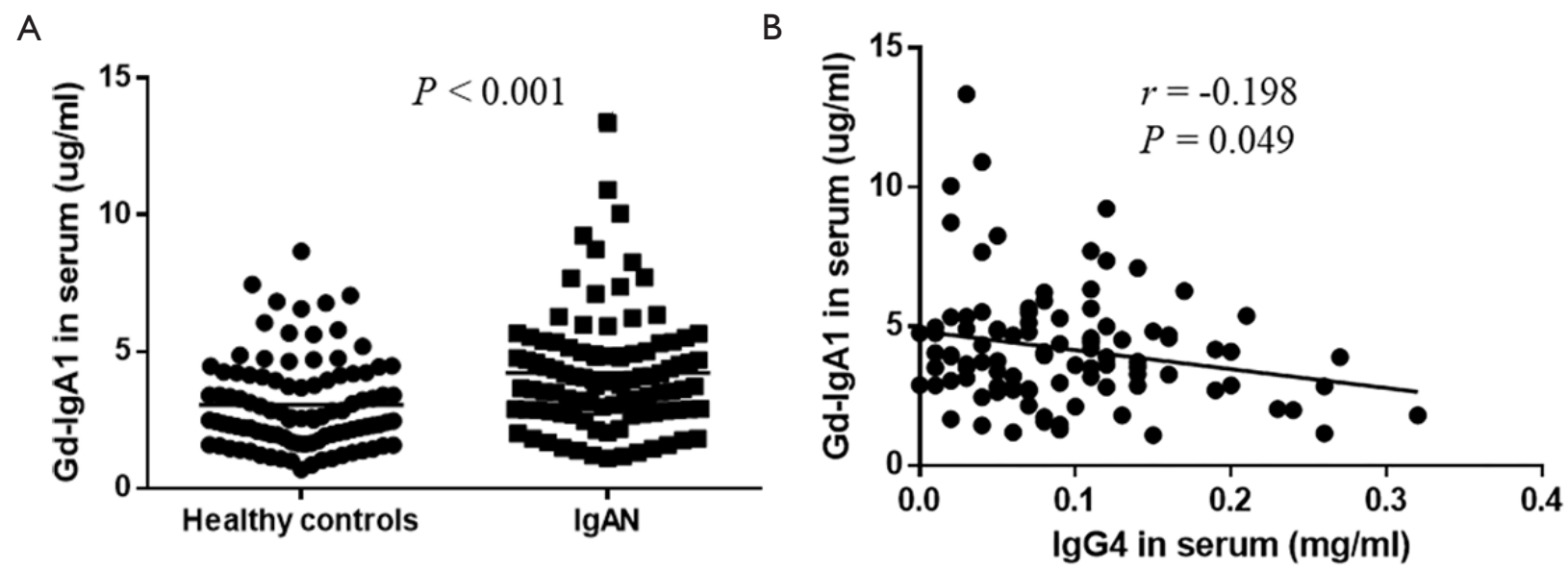

Figure S3 Increased serum Gd-IgA1 concentrations and its correlation with serum IgG4 levels in IgAN. (A) Serum Gd-IgA1 concentrations in IgAN were higher than that of healthy controls. (B) IgG4 was negatively correlated with Gd-IgA1 in IgAN. IgAN, IgA nephropathy. GdIgA1, galactose-deficient IgA1. 